\section{L. d'Agostino'}

\author{
A. J. Acosta \\ California Instltute of Technology, \\ Pasadena, CA 91125
}

\section{A Cavitation Susceptibility Meter With Optical Cavitation Monitoring-Part Two: Experimental Apparatus and Results}

This work is concerned with the development and operation of a Cavitation Susceptibility Meter based on the use of a venturi tube for the measurement of the active cavitation nuclei concentration in water samples as a function of the applied tension. The pressure at the venturi lhroat is determined from the upstream pressure and the local flow velocity without corrections for viscuus effects because the flow possesses a laminar potential core in all operational conditions. The detection of cavitation and the measurement of the flow velocity are carried out optically by means of a Laser Doppler Velocimeter. A custom-made electronic Signal Processor is used for real time data generation and temporary storage and a computerized system for final data acquisition and reduction. The implementation of the whole system is described and the results of the application of the Cavitation Susceptibility Meter to the measurement of the water quality of lap water samples are presented and critically discussed with reforence to the current state of knowledge on cavitation inception.

\section{Introduction}

Experience shows (Knapp et al., 1970) that the maximum tensile stress that liquids can theoretically sustain according to thermodynamic considerations is much larger than observed in practice. It has therefore been postulated that the tensile strength of liquids is considerably reduced by the presence of weak spots, generically called "nuclei," which act as prefcrential points for the onset of liquid rupture. The concentration and susceptibility of nuclei profoundly affect the inception, development, and scaling of cavitation in a wide variety of technically important applications. Therefore significant efforts have been made to develop effective cavitation nuclei detection methods (Billet, 1986; Billet, 1985; Oldenziel et al., 1982; Godefroy et al., 1981). Cominonly used techniques, like Coulter counters, acoustical attenuation, acoustical and optical scattering, photography and holography, monitor noncavitating liquids and therefore cannot provide reliable information on cavitation nuclei susceptibility. Cavitation Susceptibility Meters (CSM's) are intended to overcome this limitation by directly measuring the active nuclei concentration as a function

\footnotetext{
${ }^{1}$ Now at the Dipartimento di Ingegnẹria Aerospaziale, Università di Pisa, 56126, Pisa, Italy.

Contributed by the Fluids Engineering Division for publication in the JourNal of FlUIDS ENGIIEERING. Manuscript received by the Fluids Engineering Division July $20,1989$.
}

of the applied tension in a flow through a small venturi tube, where cavitation is induced under carefully controlled conditions. In the original design by Oldenziel (Oldenziel, 1982a; Oldenziel 1982b) a glass venturi is used and cavitation is detected optically. Later applications employ stainless steel venturi tubes, where cavitation bubbles are detected acoustically (Lecoffre and Bonnin, 1979; Le Goff and Lecoffre, 1983; Shen et al., 1984). Among the advantages of CSM's over alternative techniques are the absence of resolution limitations in the minimum size of nuclei they can detect and the relative convenience of data analysis. On the other hand, CSM's are subject to a number of unwanted phenomena, like flow separation, nuclei interference, clooking and surface nuclei effects, which severely limit their performance. This paper is part of a systematic investigation of CSM's and critically describes the implementation and operation of the CSM recently developed at the California Institute of Technology (d'Agostino and Acosta, 1983; d'Agostino, 1987; d'Agostino et al., 1989). The principles of operation and the main considerations leading to the current design have been reported in a companion publication (d'Agostino and Acosta, 1991).

\section{Experimental Apparatus}

The design of the CSM described herein is based on the indications of a detailed operational analysis previously re- 


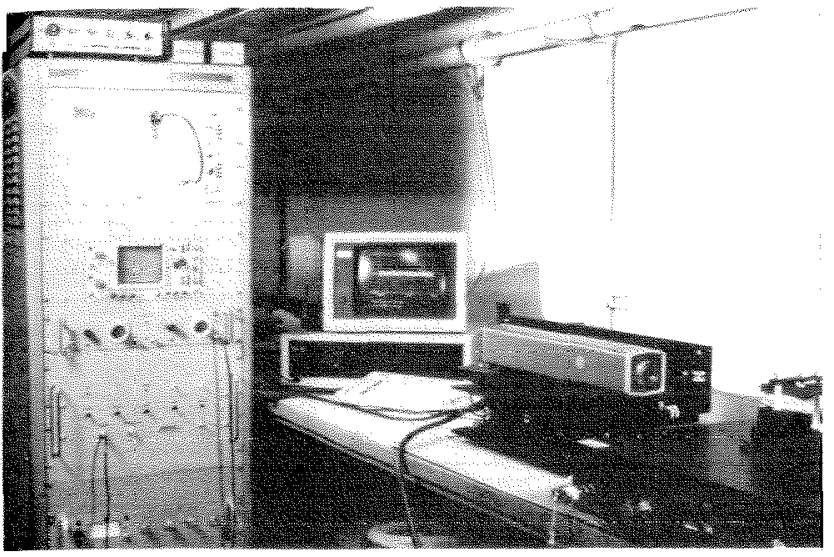

Fig. 1 General view of the CSM experimental apparatus. In the foreground on the right: the lubeams supporting the laser and the baseplate where most of the optical and fluidic components are mounted. In the background: the electronic instrumentation rack (left) and the data acquisition computer (center).

ported in earlier works (d'Agostino and Acosta, 1983; d'Agostino, 1987) and more recently summarized in a companion paper (d'Agostino and Acosta, 1991). The results of this analysis suggested to develop a CSM where cavitation occurs in the restricted section of a transparent venturi tube. The throat velocity is measured by a back-scattering Laser Doppler Velocity (LDV) and the upstream pressure by an absolute pressure transducer. The LDV signal is also used to detect the occurrence of cavitation at the throat of the venturi tube. The pressure gradient in the venturi throat is ideally zero and thus the slip velocity between the bubbles and the liquid is also zero. Cavitating bubbles are, therefore, accurate velocity indicators. The throat pressure is controlled by adjusting the exhaust pressure and is calculated from the throat velocity and the upstream pressure using Bernoulli's equation for ideal, incompressible, steady, fully-wetted flow, without corrections for viscous effects because the flow possesses a laminar potential core in all operational conditions. The dependence of the active nuclei concentration on throat pressure is measured by repeating the procedure at different exhaust pressures.

A general view of the experimental apparatus is shown in Fig. 1. The electronic instrumentation rack is on the left, the data acquisition and reduction computer is in the center and the optical and fluidic components of the CSM are in the foreground on the right. The connections of the various parts comprising the CSM are illustrated in Fig. 2. The water from

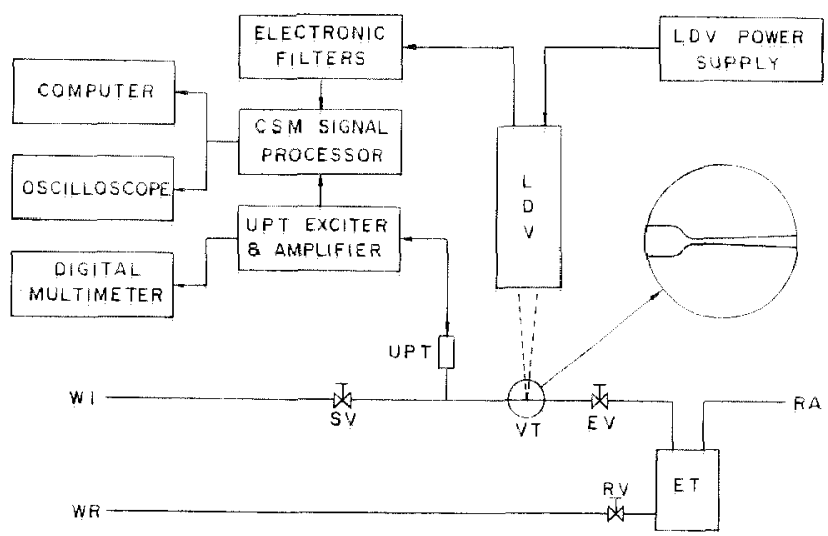

Fig. 2 Schematic of the various components of the CSM experimental apparalus: water inlet (WI), sampling valve (SV), upstream press ure trans. ducer (UPT), venturi tube (VT), exhaust valve (EV), exhaust tank (ET), regulated air pressure line (RA), return valve (RV), water return (WR), las er Doppler velocimeter (LDV).

the water inlet (WI) passes through the sampling valve (SV), the venturi tube (VT), the exhaust valve (EV) and is finally collected in the exhaust tank (ET). The pressure in the exhaust tank is kept constant by the regulated air pressure line (RA), which is used to control the flow rate and therefore the pressure generated at the throat of the venturi. The static pressure of the sampled water is measured by a pressure transducer (UPT) located upstream of the test venturi. Periodically the sampled water is removed from the exhaust tank through the return valve (RV) and the water return line (WR) by increasing the regulated air pressure. During test runs the dual beam backscattering Laser Doppler Velocimeter (LDV) monitors the occurrence of cavitation and the flow velocity at the throat of the transparent venturi (VT). The analysis of the signals from the LDV and the upstream pressure transducer is carried out by an especially designed electronic Signal Processor for real time generation and temporary storage of the relevant data. The LDV generates a burst when an inhomogeneity such as a cavity or a suspended particle scatters light during its motion through the focal point. After band-pass filtering this burst ideally consists of a Doppler carrier frequency modulated by a Gaussian-shaped envelope. The Doppler frequency is proportional to the velocity of the scatterer. The amplitude of the burst's envelope is instead mostly related to the scatterer size, although it also depends in a complex way on its shape, optical

\section{Nomenclature}

$A=$ cross-sectional area

$C_{c}=$ venturi contraction coefficient

$C_{e}=$ venturi expansion coefficient

$D=$ venturi diameter

$f=$ probability density distribution

$L=$ length

$M=$ number of data groups

$n\left(p_{i}\right)=$ concentration of nuclei with critical pressure not smaller than $p_{t}$

$N=$ integer number

$N_{b}=$ number of cavitation events or bubbles

$N_{z c}=$ number of zero crossings

$p=$ pressure $p_{t}=$ venturi throat pressure

$p_{u}=$ venturi upstream pressure

$q=$ volume flux

$R=$ venturi radius

$t=$ time

$t_{s}=$ sampling time

$T=$ temperature

$T_{b}=$ arrival time of a cavitation event or bubble

$T_{\mathrm{g}}=$ Doppler gate time

$T_{p}=$ arrival time of a velocity tracer or particle

$\boldsymbol{u}=$ velocity

$u_{t}=$ venturi throat velocity

$\alpha=$ air content

$\delta=$ boundary layer thickness

$\epsilon=$ error

$\bar{\nu}_{b}=$ average cavitation event rate $\sigma=$ standard deviation

$\sigma^{2}=$ variance

Subscripts

$b=$ bubble or cavitation event

$c=$ venturi tub contraction

$\boldsymbol{e}=$ venturi tube exhaust

$o=$ reference conditions

$p=$ particle or velocity tracer

$s=$ sample

$t=$ venturi tube throat

$u=$ upstream

$z c=$ zero crossing

Acronyms

CSM = Cavitation Susceptibility Meter

LDV = Laser Doppler Velocimeter 


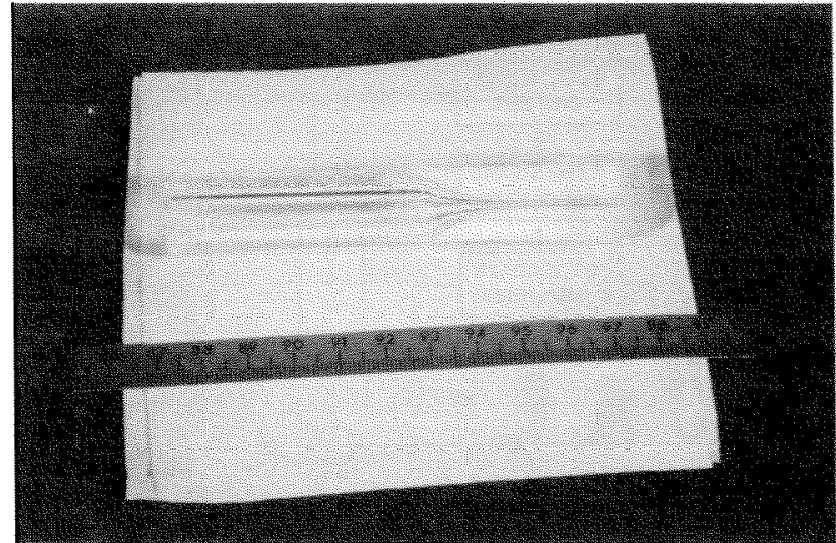

Fig. 3 Close-up view of the CSM venturi tube No. 1. A cylindrical shell of cast transparent resin contains the glass blown venturi tube for me. chanical protection and easy installation. The coniraction ratio of the tube is about $1 / 100$, the throat diameter is about $1 \mathrm{~mm}$ and the exit diameter is about $1.2 \mathrm{~mm}$.

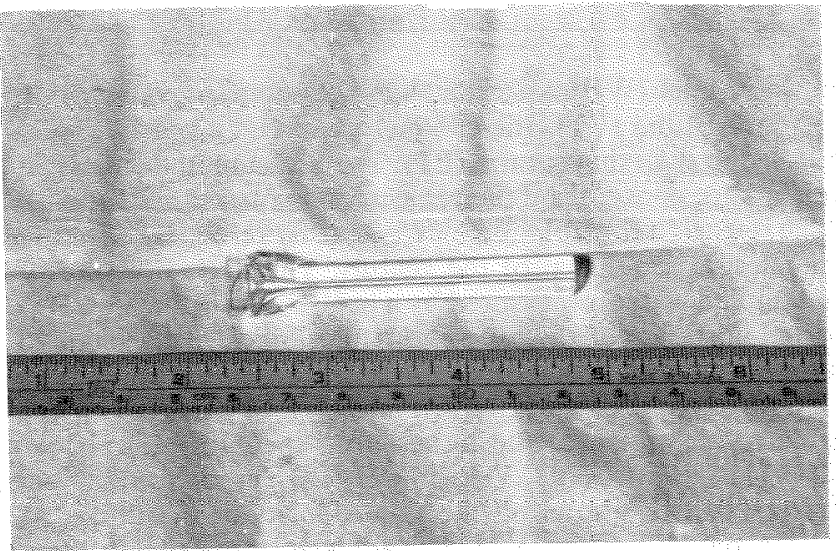

Fig. 4 Close up view of the CSM venturi tube No. 2. This blown glass venturi has thicker walls, a contraction ratio of about $1 / 100$, a throat diameter of about $1 \mathrm{~mm}$, a long straight section after the diffuser and an exhaust diameter of about $1.2 \mathrm{~mm}$.

properties and on the location of its trajectory through the LDV focal point. The CSM Signal Processor uses the intensity and the Doppler modulated frequency of the LDV bursts to respectively monitor the occurrence of cavitation and to measure the flow velocity. The instantaneous upstream pressure of the water is provided by the output of the pressure transducer. At the conclusion of each run the data are transferred to the minicomputer for final acquisition, storage and reduction. The LDV signal is normally monitored by an oscilloscope and the signal of the upstream pressure transducer by a digital multimeter.

Two types of glass venturi tubes have actually been used. The first type, indicated as tube No. 1, is an extremely fragile blown glass venturi contained in a cylindrical shell of transparent acrylic resin (see Fig. 3) for mechanical protection and connection to the hydraulic lines. The second type of glass venturi, indicated as tube No. 2, has much thicker walls and can therefore be directly connected to the hydraulic lines with. out mechanical protection. It is shown in detail in Fig. 4. These two venturi tubes have very similar fluid mechanical characteristics. They both have about the same throat diameter $\left(D_{t}\right.$ $\simeq 1 \mathrm{~mm})$, the same throat section length $\left(L_{t} \simeq 5 \mathrm{~mm}\right)$ and the same geometrical contraction and expansion ratios $\left(C_{c} \simeq\right.$ $1 / 100$ and $C_{\varepsilon} \simeq 1.44$, respectively). Hence, the velocity profile determined from fully wetted flow measurements with artificial seeding using the LDV is also very similar in the two venturis and the corresponding minimum pressure developed is in both cases $p_{t_{\min }} \simeq-35 \mathrm{kPa}$. The most important fluid mechanical
BLOCK DIAGRAM OF C.S.M. INFORMATION FLOW

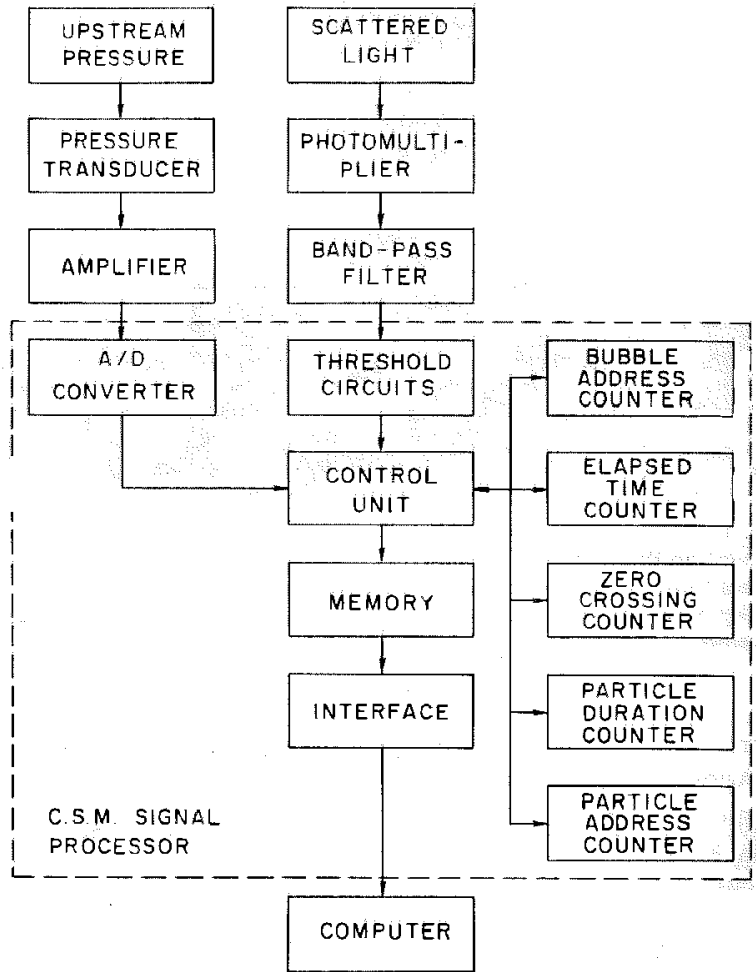

Fig. 5 Block diagram of the information flow in the CSM Signal Pro: cessor

difference between the two venturi tubes is the different length of the exit section, much shorter in the venturi No. 1 (only a few $\mathrm{mm}$ ) than in the venturi No. 2 (about $3 \mathrm{~cm}$ ). As a consequence, the flow in the second venturi is considerably more stable with respect to the insurgence of cavitation-separation.

\section{Data Acquisition and Reduction}

A simplified block diagram of the CSM signal processing and data acquisition is shown in Fig. 5. The output of the photomultiplier is band-pass filtered for separating the Doppler frequency from the electronic noise and sent to the CSM Signal Processor, where it is amplified before entering the threshold circuits. Here a zero level and two couples of adjustable, symmetric threshold levels are used to reject the residual noise and to discriminate valid Doppler bursts coming from a velocity tracer from the ones coming from a cavitation event. For simplicity cavitation events will be indicated as bubbles and velocity tracers as particles, although in practice smaller cavities probably represent the majority of the velocity tracers recognized by the CSM Signal Processor during a typical CSM run. The information from the threshold circuits is used by the Signal Processor to control five counters and to generate, collect and temporarily store in real time the following data:

- when a cavitation event (bubble) is recognized:

1 the occurrence time measured from the beginning of the run (bubble elapsed time, $T_{b}$ );

- when a velocity tracer (particle) is recognized:

1 the occurrence time measured from the beginning of the run (particle elapsed time, $T_{p}$ );

2 the duration of the Doppler burst (particle gate time, $T_{\mathrm{g}}$ );

3 the number of zero crossings of the Doppler burst $N_{z c}$;

4 the upstream water pressure $p_{u}$;

A maximum of 1024 cavitation bubbles and velocity tracers can be independently recorded at a maximum acquisition rate 
of about 10000 events per second. $\Lambda t$ the conclusion of each run the data collected by the CSM Signal Processor are serially transferred to the microcomputer for final acquisition, storage on a magnetic disk and reduction.

The data reduction develops, through the following steps:

1 zero crossing validation and statistical filtering of the Doppler frequency and upstream pressure data;

2 computation of the average potential core velocity, of the potential core velocity data standard deviation and of the boundary layer thickness;

3 computation of the average throat pressure and of the throat pressure data standard deviation;

4 validation of the arrival times of cavitation events and velocity tracers;

5 computation of the observed and expected occurrence frequency distributions of the delay times between cavitation events;

6 computation of the unstable nuclei concentration and of its standard deviation.

During the first step Doppler frequency data are computed by dividing the number of zero crossings of each burst by twice its duration. To reject spurious bursts, only counts with a preselected minimum number of zero crossings are used (zero crossing validation). Both the Doppler frequency data and the upstream pressure data contain a relatively small number of outliers due to various noise sources in the electronics. In addition, the Doppler frequency data may sometimes contain a significant number of low frequency readings from scatterers deep inside the venturi boundary layers. To eliminate the noise and to isolate the velocity information primarily coming from the venturi's potential core the Doppler frequency data are statistically filtered by only retaining those readings whose deviation from their average values does not exceed a preset multiple of their standard deviation (usually three standard deviations). The same procedure is also applied to the upstream pressure data, leading to the determination of the average upstream pressure $\bar{p}_{u}$ and of the upstream pressure data standard deviation $\sigma_{p_{u}}$.

The computation of the average potential core velocity, of the potential core velocity data standard deviation and of the boundary layer thickness (step 2) is carried out as follows. First the observed occurrence frequency distribution of the measured velocity data is calculated in the form of a histogram chart. When boundary layer effects are important and their role is not masked by other factors, this distribution is negatively skewed and therefore its third central moment is negative. If this is not the case the expected value and the standard deviation of the obscrved distribution are simply used to compute the average potential core velocity $\bar{u}_{t}$ and the standard deviation of the potential core velocity data $\sigma_{u_{f}}$, while the boundary layer thickness $\delta$ is taken to be zero. Otherwise, assuming uniformly distributed scatterers throughout the venturi cross-section, a theoretical probability distribution of the mcasured velocity is derived that parametrically depends on $\bar{u}_{t}, \sigma_{u_{t}}$ and the ratio of the boundary layer thickness to the local duct radius $\delta / R_{t}$. These parameters are then determined by fitting the theoretical distribution to the observed one. This process results in a slightly higher estimate of the average throat velocity and in a small reduction of the standard deviation of the throat velocity data depending on the value of the parameter $\delta / R_{i}$.

The venturi throat pressure $p_{t}$ (step 3 ) is deduced from the measurements of the upstream pressure $p_{u}$ and of the throat velocity $u_{t}$ using Bernoulli's equation for ideal, incompressible, steady, fully wetted flow. An unavoidable problem associated with this technique is that the throat pressure, being inherently small compared to the upstream pressure and the kinetic pressure drop, is expressed as the difference of two almost equal quantities. Thus, small relative errors in the evaluation of thesc quantities lead to a much larger relative error for the throat pressure. The statistical analysis for the determination of the average value $\bar{p}_{t}$ and the standard deviation $\sigma_{p_{t}}$ of the throat pressure data $p_{t}$ is carried out assuming that the two independent data populations $p_{u}$ and $u_{t}$ are normally distributed about their average values $\bar{p}_{u}$ and $\bar{u}_{t}$, with variances $\sigma_{p_{u}}^{2}$ and $\sigma_{u}^{2}$, respectively,

Clearly, the arrival times of LDV bursts are monotonically increasing and, in steady conditions, approximately proportional to their index. These properties are used to validate the data and to assess the uniformity of both the sample and the test conditions. First, the linear regression curve of the arrival times as a function of their index is calculated. Then, the data which are not in monotonically increasing order and whose deviation from the regression line is the largest are eliminated, in order to eliminate spurious readings (step 4).

The observed frequency distribution of the time $t$ between successive cavitation events is calculated and a histogram chart is constructed by sorting the $N_{b}$ data in, say, $M$ groups equally spaced in time for comparison with the expected Poissonian exponential distribution $f(t)=N_{b} \bar{v}_{b} e^{-\bar{v}_{b}}$, where $\bar{\nu}_{b}=N_{b} / t_{s}$ is the average arrival rate of cavitation events duing the sampling time $t_{s}$ (step 5). Finally, the volume flow rate $q=A_{i} \bar{u}_{i}$ is used to estimate the unstable nuclei concentration $n\left(p_{t}\right)=$ $N_{b} / t_{s} q$ and its standard deviation $\sigma_{n}=\sqrt{N_{b}} / t_{s} q$ (step 6).

\section{Experimental Procedure, Results, and Discussion}

The calibration of the CSM system has been carried out using a mercury barometer for the upstreanl absolute pressure transducer and a rotating disk in air as a source of a finely controlled velocity field for the LDV. The overall accuracy (linearity plus repeatability) of the calibrations was better than $200 \mathrm{~Pa}$ for the upstream pressure transducer over the range 20 to $160 \mathrm{kPa}$, and $0.05 \mathrm{~m} / \mathrm{s}$ for the LDV over the range 9 to 22 . $\mathrm{m} / \mathrm{s}$. Thus, in general, the CSM instrumentation error was always negligible with respect to the inherent dispersion of the measured quantities.

The experimental procedure for measuring the water quality with the CSM is relatively simple. The water source is connected to the CSM test section with the shortest possible tubing, taking care to avoid abrupt changes of the duct internal section. An overflow line is also used to reduce as much as possible the transfer time of the sample to the CSM. The temperature of the sampled water is measured with a thermometer and its air content with a manometric van Slyke meter before each CSM run.

The location of the LDV focal point is very important for obtaining high quality repeatable results from the CSM. Experience showed that the best location is at the end of the venturi throat section. This choice provides better LDV signal strength, due to the larger size of the scattering bubbles, with acceptable dispersion of the velocity data. The electronic settings also are of crucial importance for the operation of the CSM, since they determine the number of LDV bursts recognized and counted as cavitation events. Proper choice of these settings depends on the correlation of the scatterer size to its LDV signature and on the definition of a general criterion for discriminating in dynamic conditions unstable cavitating nuclei from stable ones. This first aspect can be partially addressed by calibrating the LDV signal from cavities of known sizes, for example by monitoring the sampled water with holographic methods (d'Agostino et al., 1989; d'Agostino and Green, 1989). The discrimination of unstable cavitating nuclei, however, is essentially equivalent to the definition of the cavitation inception conditions, which is still an open problem in cavitation research. Until these two problems are satisfactorily solved, the selection of the electronic settings of the CSM remains, at least to some extent, arbitrary. In the present case the electronic settings were chosen trying to optimize the re- 


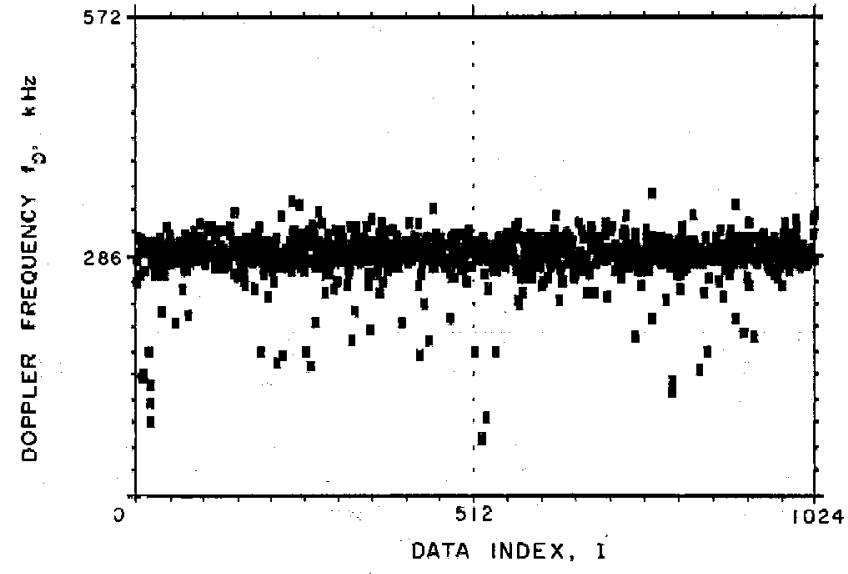

Fig. 6 Doppler frequency data $t_{0}$ as a function of the data index / in a typlcal CSM run. The data refer to a tap water sample with initial pressure $\rho_{0}=1 \mathrm{alm}$, temperature $T=21^{\circ} \mathrm{C}$, air contenl $\alpha=20.5 \mathrm{ppm}$, throat pressure $p_{t}=-15 \mathrm{kPa}$ and velocity $u_{t}=14.8 \mathrm{~m} / \mathrm{s}$.

sponse of the electronics and to ensure the applicability of the same settings to the whole range of the expected operational conditions.

The results reported in this section have been obtained testing tap water in the venturi tubes No. 1 and No. 2 briefly described in the previous section. Three different flow regimes have been observed:

- travelling bubble cavitation;

- cavitation-separation and sheet cavitation;

- spot and resonant cavitation.

Clearly only the first one is the nominal operational regime of the CSM where mcaningful water quality measurements can be made. The others involve unwanted phenomena, which often perturb the flow conditions in an uncontrollable way and prevent the possibility of reliably measuring the active cavitation nuclei concentration in the sampled water. Therefore, for conciseness, they will not be examined here.

The traveling bubble flow regime is characterized by the random occurrence of cavitation bubbles in the CSM venturi and has been observed in both venturi tubes No. 1 and No. 2. The bubbles start to develop in the bulk of the liquid in the upstream region of the venturi's throat section, travel downstream reaching their maximum size somewhere in the diffuser or sometimes in the exit section, and later collapse. Under stroboscopic light the bubbles appear approximately spherical, at least during their growth phase. The length of the region where cavitation bubbles can be observed, their maximum size and occurrence rate depend on the flow conditions, clearly increasing with the throat tension and the velocity of the flow. In the venturi tube No. 2 (the only one where the entire life cycle of the cavitation bubbles can be observed) the length of the cavitation region ranges from about 5 to $25 \mathrm{~mm}$. The maximum size of the bubbles varies greatly and can even be comparable to the local diameter of the venturi. Optical observation also indicates that larger bubbles occur when the cavitation event rate is relatively low. This behavior suggests the possible presence of significant interactive effects among the bubbles at high concentrations and is directly related to the general problem of flow saturation, which will be discussed later.

Some of the physical raw data and of the reduced data from a typical CSM run of a tap water sample with an air content of $20.5 \mathrm{ppm}$ at $21^{\circ} \mathrm{C}$ are shown in Fig. 6 through Fig. 8. The water sample, initially at atmospheric pressure, was tested in the venturi tube No. 2 at a throat pressure of $-15 \mathrm{kPa}$ corresponding to a velocity of $14.8 \mathrm{~m} / \mathrm{s}$.

The Doppler frequency data in the first plot of Fìg. 6 are centered about their average value indicated on the scale. The

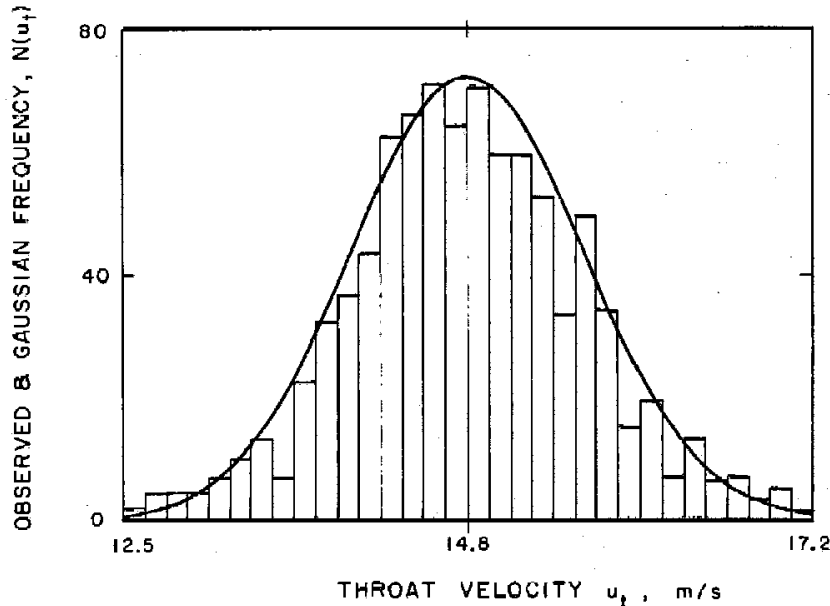

Fig.7 Observed distributlon of filtered throal velocity data (histograms) compared to the Gaussian distribution (solid line) of equal mean and standard deviation for the data sample of Fig. 6

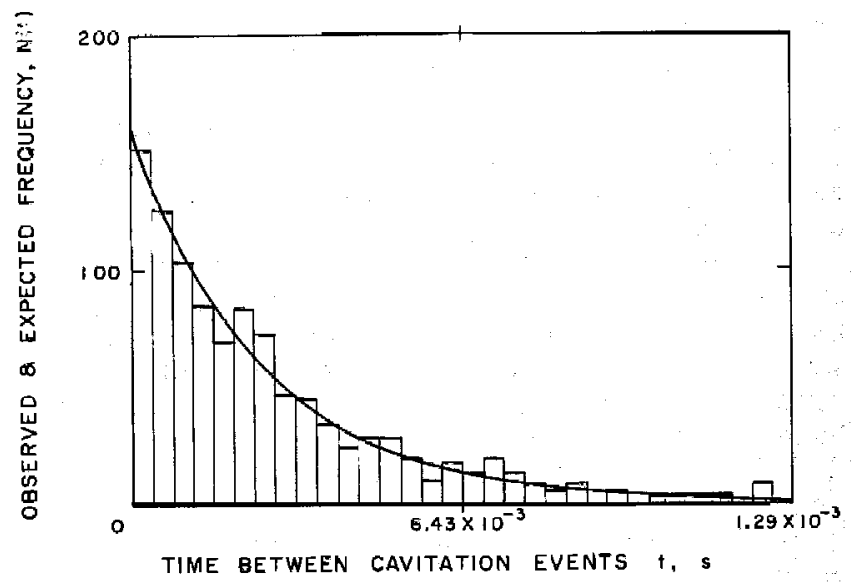

Fig. 8 Obsetred distribution of time intervals between cavitation events (histograms) in a typical CSM run compared to the theoretical exponentlat distribution (oolid line) of equal average arrival rate. The data refer to the tap water sample of Fig. 6

dispersion of the data is mainly due to the intrinsic slightly unsteady nature of the flow and to the non-uniformity of the velocity profile in the venturi. However, this data set also contains a small but significant number of low frequency readings. Some of these readings do not appear to be randomly distributed during the run, but rather seem to occur sequentially in small groups. Experience with the operation of the CSM indicates that they are probably coming from cavities rather than, for example, from small particles normally present in the boundary layer. Most likely they are due to bubbles originating from the disintegration of small attached cavities from surface nuclei under the action of the incoming flow and later swept downstream through the LDV probe volume before they had time to accelerate to the surrounding flow velocity. This conclusion is also supported by the observation that cavitation events occasionally occur in clusters, which correspond to short, almost horizontal stretches in the plot of the bubble arrival time data.

In the second plot (Fig. 7) the histogram chart representing the velocity data distribution is compared to the Gaussian curve of equal average value and standard deviation. Readings deviating from the mean more than three standard deviations have been eliminated. Note that the observed distribution of velocity data seems to follow rather closely a normal distribution and does not display any appreciable skewness which could be attributed for example to the effects of the boundary layers, as previously mentioned. Experience from other runs 


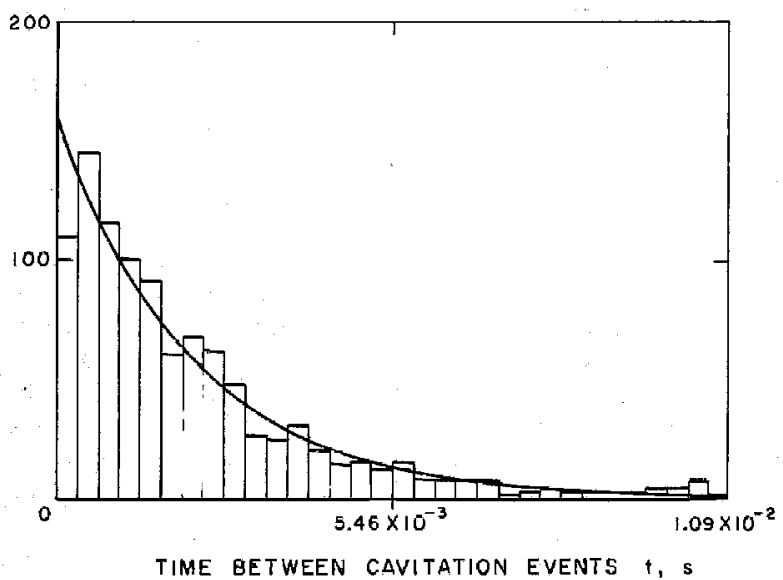

Fig. 9 Observed distribution of time intervals between cavitalion events (histograms) slightly deviating from the theoretical exponential distribution (solid line) as a consequence of short range bubble interference effects

showed that the skewness of velocity distributions is usually small in both directions and probably statistically insignificant, as if the biasing effects of the boundary layer were unimportant or masked by sume other phenomenon. Therefore the estinates of the ratio of the boundary layer thickness computed from the observed negative skewness of the velocity data distribution should be regarded with some skepticism, despite the fact that they never gave unrealistic results.

In the last plot (Fig. 8) the histogram chart representing the distribution of the observed time intervals between cavitation events is compared to the Poissonian exponential distribution expected to describe the occurrence of cavitation when the flow conditions are constant and the cavitation events are uncorrelated. This comparison provides a way to assess the importance of short range nuclei interference effects at the throat of the CSM venturi due to the inhibiting action that the pressure perturbations from a growing cavity can exert on the growth of neighboring nuclei. Clearly, this kind of interaction especially penalizes the occurrence of the shortest time intcrvals between successive cavitation events with respect to the theoretical Poissonian distribution. The data from Fig. 8 do not show any evidence of important short range bubble interference. For comparison, an example of a distribution deviating from the expected exponential behavior as a consequence of nuclei interference effects is shown in Fig. 9 for a different CSM run. In general these effects in CSM flows are not large. At most they affect a few percent of the total number of cavities and only tend to appear at heavier cavitation rates, when the separation between cavitating nuclei is proportionally reduced. This is not surprising when considering that the minimum average separation of cavitation bubbles is comparable to the length of the CSM venturi tube even at the highest cavitation rates. Clearly, more cavities are actually present in the flow whose LDV signatures are not large enough for them to be recognized as cavitation events by the CSM Signal Processor. For these cavities short range interference effects are likely to be more significant.

When a large number of cavities is continuously present in the cavitation region of the CSM venturi the collective effects of the bubble volume changes produce a global, permanent increase of the pressure throughout the venturi test section (choked flow conditions). In this situation the tube is saturated and the maximum tension that venturi tube can develop at any given flow rate is reduced, as it will be shown later in this section. However, the pressure experienced by any one bubble is not appreciably moditied by the presence of the neighboring ones, since saturation is a large scale effect due to the collective contribution of many cavities widely distributed in the flow.

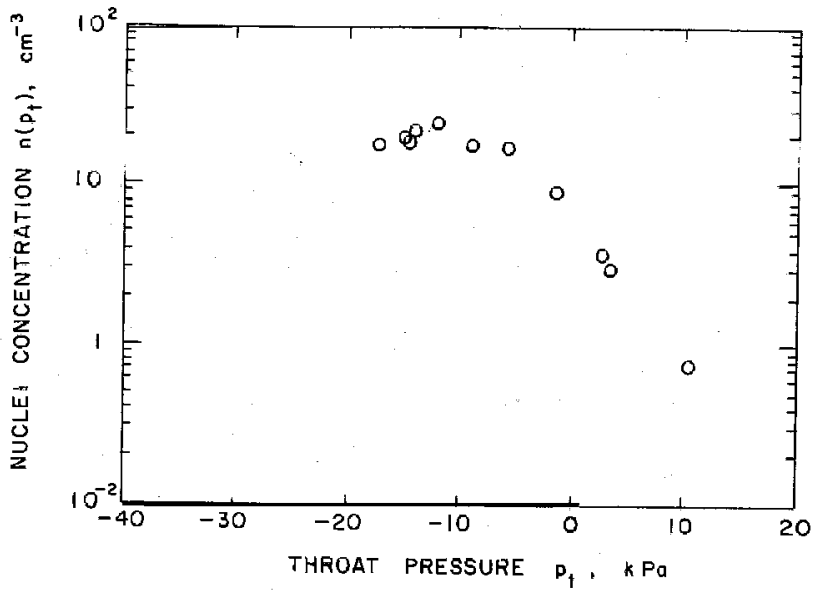

Fig. 10 Cavitating nuclei concentration $m(p)$ measured by the CSM as a function of the venturi throat pressure $p_{t}$ in a sample of tap water with temperature $T=21^{\circ} \mathrm{C}$ and air content $\alpha=20.5 \mathrm{ppm}$

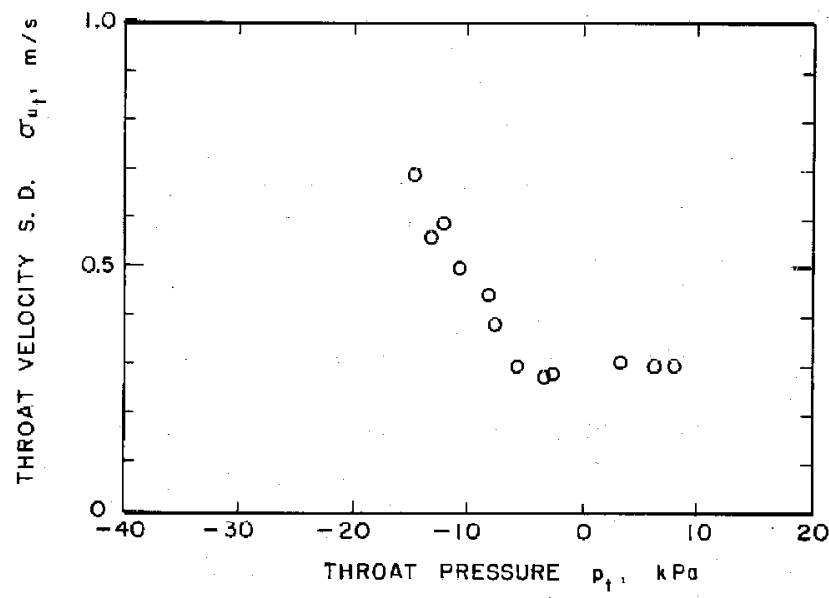

Fig. 11 Standard deviation of the throat velocity data $\sigma_{u}$ as a function of the throat pressure $p_{t}$ in the tap water sample of Fig. 10

Therefore this kind of bubble interference will affect the average cavitation rate but will not be reflected in an anomalous distortion of the observed distribution of the time intervals between cavitation events. Clearly, saturation phenomena tend to increase with the concentration of unstable cavitation nuclei in the water sample and with the tension they are exposed to and impose limitations to the range of liquid quality measurements which can be carried out with the CSM.

An example of the application of the CSM to the measurement of the cavitation nuclei concentration as a function of the venturi throat pressure by means of repeated runs on a sample of tap water at $21^{\circ} \mathrm{C}$ with a dissolved air content of $20.8 \mathrm{ppm}$ is shown in Fig. 10 and Fig. 11. These results are generally representative of CSM water quality measurements on other tap water samples in similar conditions. At first the concentration of cavitation nuclei increases about exponentially with the applied tension, as also reported by other investigators (Oldenziel, 1982a; Shen and Gowing, 1985; Shen et al., 1986). When the throat pressure is further reduced below about $-7 \mathrm{kPa}$ the concentration of cavitation nuclei reaches a maximum ranging from 20 to $40 \mathrm{~cm}^{-3}$ and remains nearly constant thereafter. The observed behavior of the concentration of cavitation nuclei probably reflects the actual lack of cavitation nuclei which become active when the throat pressure is lowered below about $-7 \mathrm{kPa}$. In this case a further decrease of the throat pressure would simply produce a more violent growth and collapse of the available population of active nuclei. Larger perturbations would then be induced in the flow, 
thus generating the parallel increase of the velocity data stand: ard deviation from a previously constant value that is clearly apparent in Fig. 11. This Interpi tation has also been fuvorably tested by comparison of CSM results with holographically determined nuclei concentration densicy distributions (d'Agostino et aj, 1989; d'Agostiso and Green, 1989). Finally, note that, assurning in first approxinnation that the throat pressure is correctly measured also in the presence of heavy cavitation, tive In:ninum pressure generated by the CSMM venturi in the run of Fig. 10 is of the order of $-15 \mathrm{kPa}$ and therefore conslderably higher than the venturi cun develop in the absence of extensive cavitation. It appears that saturation phenomena are responsible for the observed decrease of the maximum achievable tension in the venturi and the consequent performance limitatjons.

At given settings of the CSM the accuracy in the deternination of the water quality is due to the errors in the measurement of the utvitating nuclei concentration and of the average throat pressure. With good approxiraation the throat pressure data are hormally distributed and the occurrence of cavitating nuclei is a Poisson process, provided that the flow conditions are conseant during the run. Then, both the above errors are inversely proportional to the squarc root of the size of the data sarnple (Browlee, 1960) and therefore can be significantly reduced by averaging the measured quantities on a sufficiently large number of data. Since the number of fijtered data is usually close to 900 , the relative $r, m, s$. error of the measurement of the cavitating nuslei concentration is tather small, of the order of a few percent. The standard deviationl of the throat pressure data is quite large in relative sense due to the inherent dispersions of the throas velocity data and to the small value of the pressure at the CSM venturi throat. Values of about 5 to $10 \mathrm{kP}$ a for the standard deviation of the throat pressuse data are representative of the results usually obtained from CSM runs. As mentioned earlier, this value must be divided by the square root of the sample bize in order to obtain the standard deviation of the average throat pressure. With the sane size of data sanple as proviously assunred the corresponding error in the determination of the average throat pressure is therefore of the urder of a few hundred Pascals. The above expected errors are both relatively small, but it should be born in misul that they strictly refer to the measurement procoss alone. In a broader sensc the indetermination in the measurement of the water quality also includes the effects of the uncertuinties in the relationship between the LDV jignal intensity and tire stze of cavitating bubbles and in the discrinination of unstable ubclel from the stable ones. The former of these effects is related to the specific nuclei detection technique used in the CSM and could presumably be resolved to some extent by alibratilg the instrument response with cavities of known sizes, as previously suggested. The latter is instead shared by all currently devlsed techniques of cavitation nuclei detection. The inclusion of all error sources involved Inakes the measurement of the liquid quality with CSM's (and with other alternative methods) considerably more uncer tain than it would first appear from the above considerations.

\section{Conclusions}

The following conclusions cau be drawn from the available experience on the CSM systetu at the present stage of the project:

- three difteren flow regines have been observed in the CSM venturi tube:

1. travelling bubbie cavitation (during nomal opetation);

2 cavitation-separaion and sheet cavitacion;

3 spot and resuluas uavitation.

- the proposed dcsign concept has beell successfully dernonstrated in all its compolients;

- the CSM is able to measure the concentration of active cavitation nuclei as a function of the throat pressure over an exlended range of applieal tensions;

- the maximun yalue of the tension currently attainable $(-35$ $\mathrm{kPa}$ ) is otien insufficient fur cavitating waters with relaţively low nuclei cunteni;

- at high nuclei concentrations flow saturation produces a generalized increase of the pressure and a parallcl decrease of the velocity througlout the venuri throat sectlon and therefore limits the performance of the CSM by reducing the maximum value of the tension exerted on the liquid;

- contrary to preliminary expeclations, the arrival of cavitation events remains nearly Poissonian alșo at high cavitation results. Therefore saluration does not produce important short range interfcrence effects berween cavitating nuclei nor, consequently, poses significant limilations to the measurement of the concentration of active cavitation nuslei other than those due to the reduction of the maximum tension applied to the liquid;

- the maximun measurable concentration of active cavitation nuclei in the water samples tested so far appears to be limfted by the available stupply of nuclei in the sarnples rather than by the occurrence of sacuration;

- active cavitation nuclei concentrations up to about $40 \mathrm{cal}^{-3}$ have been recorded with little evidence of short range it:terference effects between cayitating bubbles;

- the flow is very sensitive to laminar separation in the diffuser, which limiss the minimun throat pressure curienty artainable to about - $35 \mathrm{kPa}$;

- cavitation-separalion and sheet cavitation frequentyy interfere with the normal operation of the CSM;

- the possibility of msasuring the velocity of individual cayities at the venturi throat represents a powerful ald unique mean for discriminating the cavitles produced by freestreaub nuclei from the ones originated fron surface nuclesi and for introducing corrections to the CSM measuremellts when necessary;

- the pussibility of recording the transit tirke of cavitating buboles is extremely useful to assess the importance of short range interference effects from the statistical propertios ot the cuvitation process;

- the measurenents of the cavitation nuclei number cuncerntration density distributions in tap water samples ubtained using the CSM show little dispersion and good repeatability;

- comparison of the CSM with a reliable nuclei detection methou (holography) is indispensable in order to "properly" chousc the elecroujc thresholds for the discrimination of cavitation events and in order to correctly interpret some of the observed results.

\section{Acknowledgments}

This research has becil funded by the Office of Naval Research and by the Naval Sea Systems Command General Hy. dromechanics Research Program administered by the David W. Taytor Naval Ship Research and Development Center. The North Atlantic Treaty Organization-Consiglio Naziunale delle Ricerche, Italy, has also contributed to the supjort of this work through a 1982 and a 1983 Fellowship for Technologioal Rescarch. Special thanks to Dr. T. T, Huang of DTNSRDC for his interest in thls work, to Mr. Jue Fontana, Mr. Elton Daly, Mr. Rich Eastvedt, Mr. Leonard Montenegro, Mr. Jolit Lee and to Miss Cecilia Lin of the Caltech staff for their assistance in the completion of the experiment and to Ds. Haskel Shapiro, Mr. Bob Kirkpatrick and their group for the design and implementation of the custom-made electronics.

\section{References}

Billet, M., 1986, "The Imporbanes and Mesturement of Catyation Nuchej,

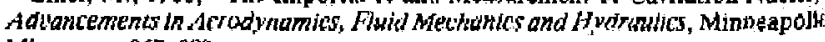
Minn., pp. 967-980. 
Billet, M., 1985, "Cavitation Nuclei Muaburemen-A Review," ASME Cavitolion and Multiphase Flow' Forum, Albuquerque, N.M., pp. 31-38.

Browlee, K. A., 1960, Statistical Theory and Methodology in Science and Engineering, Wiley,

Chahine, G. L., and Shen, Y. T., 1986, "Bubble Dynamics and Cavitation Inception in Cavitarion Susceplibility Meclers," ASME Journas of FuUus EsGLNEERINo, Vol. i08, pp. 444-452.

d'Agostino, L., 1987, "ExpcrimcntaI and Theoretical Study on Cavitation Inception and Bubbly Flow Dynamics." Ph.D. the5is, Report No. Eng. 183, 16 . Calffornia Institute of Technology, Pasadena, Calif.

d'Agostino, L., and Acosta, A. J., 1983, "On the Design of Cavitation Sugceptibility Melers," American Towing Tank Conjerence, Hoboken, N.J.

¿'Agostino, L., and Acosta, A. J., 1991, "A Cavitation Susteptibility Metser With Optical Cavitation Monitoring-Part One; Deslgn Consepts," published in this iesue pp. 261-269

d'Agostino, L., Thai Pham and Green, S., 1989, "Comparison of a Cavitation Susceptibility Meter and Holographic Observation for Nuclei Detection in Liquids" " ASME Journal of Fluivs Exgmenering, Vol. 111, No, 2, pp. 197-203. d'Agustino, L., and Oreen, S. I, 1984, "Simultaneous Cavitation Suceptibility Meter und Holographic Measurenteris of Nuclesi in Liquids," ASME Cavidation and Multiphase Flow Forum, San Diego, California, USA

Godefroy, H. W. H. E., Jansen, R. H. J., Keller, A. P., and van Renebse, R. L., 1981, "Comparison of Meaburing and Control Methods of the Water Ouainty with Respot to Caylsation Behavior," Delft Hydraulicy Laboratory Publication.

Ito $Y_{\text {, , and } O b a, ~}$, , 1980, "Cavltation Observaltons through a Fine Laser Beam Technique," Report No. 337, Institute of High Speed Mechanics, Tohoku University,
Kriapp, R. T. Daily, J, W, and Hemmitt, F. G, 1970, Cavitation, MbGraw Hill, $\mathbf{N}, \mathbf{Y}$.

Lecoffre, Y, and Bonnin, J., 1979, "Cavitation Tests and Nuvleation Control," International Sympusiam on Cauhation Inetption, New York, N. 'Y., pp. 141-145.

Le Goff, J. P., and Lecorfre, Y., 1983, "Nuclei and Cavitation," 1Ath Sympasium on Naval Hydrodynamins, National Academy Pross, pp. 215-242.

Oldenziel, D. M., 1982a, "A New Instrument in Covitation Research, the

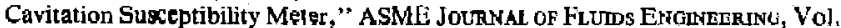
104, pp. 136-142

Oldenzitl, D. M., 1982b, "Utility of Avallable Instruments during Cavitation Tests," Proceedings of Symposium on Operatitg Problems of Pump Stations and Power Plants, JAHR, Amsterdam.

Oldenzigh, D. M., 1979, "New Instruments in Cavitation Research," International Symposium on Covitation Inception, New York, N.Y., pp. 111-124.

Oldenziel, D. M, Jansell, R. H, J., Kelle1, A. P., Lecoffre, Y., and vail Renesse, R. L., 1982, "Comparison of Instrulnents for Detection of Particles and Bubbles in Water during Cavitation Studieg," Proceedings of Symposium on Operating Problems of Pump Stations and Power Plants, IAHR, Amsterdam.

Shen, Y. T., Gowing, S., and Pierce, R., 1984, "Cavitation 3 uscepribility Metery by a Venturi," International Sympasizm on Cavitation Inception, ASME Winter Annual Meeting, pp. 9/18.

Shen, Y. T., and Gowing, S., 1985, "Swale Effects on Bubble Growth and Cavitation Inception in Cavitation Susceptibility Melers," ASME Cavitalion and Multiphuse Flow Form, Albuquerque, New Merico, pp. 14-16.

Shen, Y. T.. Gowing, S., and Ecksteln, B., 1986, "Cavitation Susceptibility Messurements of Ocean Lake and Laboratory Waters, " David W. Taylor Navai Ship Restareh and Developmient Center, Report DTNSRDC-86/D19.

\section{ADMC - Hobart, Australia, 14-18, December, 1992}

The Eleventh Australasian Fluid Mcchanics Confercnce will be held at the University of Tasmania from Monday 14, December, 1992 until Friday 18, December 1992. Papers on all aspects of fundamental and experimental fluid mechanics may be presented and published in the proceedings. Conferences in this serius are held once every three years only. Invited keynote papers will review aspects of mixing, combustion, conputational methods, hydraulics. turbomachines, hydrodynamics and occallography. Abstracts for preliminary review should be submitted to the Conference Secretariat by 31, January, 1992. These and other enquiries concerning the conference should be directed to: 11AFMC Secretariat, Department of Civil \& Mechanical Engineering, University of Tasmania, GPO Box 252C, Hobart, 7001, Australia. (Fax 002-234611). 